\title{
openheart OpenSAFELY: impact of national guidance on switching anticoagulant therapy during COVID-19 pandemic
}

The OpenSAFELY Collaborative, Helen J Curtis (D) , ${ }^{1}$ Brian MacKenna (D) , ${ }^{1}$ Alex J Walker (D) , ${ }^{1}$ Richard Croker (D) , ${ }^{1}$ Amir Mehrkar (D) , ${ }^{1}$ Caroline Morton (D) , Seb Bacon (D) , ${ }^{1}$ George Hickman, ${ }^{1}$ Peter Inglesby, ${ }^{1}$ Chris Bates (D) , ${ }^{2}$ David Evans (D) ,' Tom Ward, ${ }^{1}$ Jonathan Cockburn (D) , ${ }^{2}$ Simon Davy, ${ }^{1}$ Krishnan Bhaskaran (D) , ${ }^{3}$ Anna Schultze (D) , ${ }^{3}$ Christopher T Rentsch (D) , Elizabeth Williamson, ${ }^{4}$ William Hulme (D) , ${ }^{1}$ Laurie Tomlinson (D) , ${ }^{3}$ Rohini Mathur, ${ }^{3}$ Henry Drysdale, ${ }^{1}$ Rosalind M Eggo (D) , ${ }^{5}$ Angel Yun Wong (D) , ${ }^{3}$ Harriet Forbes (D) , 6 John Parry, ${ }^{2}$ Frank Hester, ${ }^{2}$ Sam Harper, ${ }^{2}$ lan Douglas (D) , ${ }^{3}$ Liam Smeeth (D) , ${ }^{3}$ Ben Goldacre (i) ${ }^{1}$

- Additional supplemental material is published online only. To view, please visit the journal online (http://dx.doi.org/10. 1136/openhrt-2021-001784)

To cite: , Curtis HJ, MacKenna B, et al. OpenSAFELY: impact of national guidance on switching anticoagulant therapy during COVID-19 pandemic. Open Heart 2021;8:e001784. doi:10.1136/ openhrt-2021-001784

HJC, BM, AJW and RC contributed equally.

Received 13 July 2021 Accepted 8 0ctober 2021
A) Check for updates

(C) Author(s) (or their employer(s)) 2021. Re-use permitted under CC BY. Published by BMJ.

For numbered affiliations see end of article.

\section{Correspondence to}

Dr Ben Goldacre; ben.goldacre@ phc.ox.ac.uk

\section{ABSTRACT}

Background Early in the COVID-19 pandemic, the National Health Service (NHS) recommended that appropriate patients anticoagulated with warfarin should be switched to directacting oral anticoagulants (DOACs), requiring less frequent blood testing. Subsequently, a national safety alert was issued regarding patients being inappropriately coprescribed two anticoagulants following a medication change and associated monitoring.

Objective To describe which people were switched from warfarin to DOACs; identify potentially unsafe coprescribing of anticoagulants; and assess whether abnormal clotting results have become more frequent during the pandemic. Methods With the approval of NHS England, we conducted a cohort study using routine clinical data from 24 million NHS patients in England.

Results 20000 of 164000 warfarin patients (12.2\%) switched to DOACs between March and May 2020, most commonly to edoxaban and apixaban. Factors associated with switching included: older age, recent renal function test, higher number of recent INR tests recorded, atrial fibrillation diagnosis and care home residency. There was a sharp rise in coprescribing of warfarin and DOACs from typically 50-100 per month to 246 in April 2020, 0.06\% of all people receiving a DOAC or warfarin. International normalised ratio (INR) testing fell by $14 \%$ to 506.8 patients tested per 1000 warfarin patients each month. We observed a very small increase in elevated INRs ( $n=470$ ) during April compared with January $(\mathrm{n}=420)$.

Conclusions Increased switching of anticoagulants from warfarin to DOACs was observed at the outset of the COVID-19 pandemic in England following national guidance. There was a small but substantial number of people coprescribed warfarin and DOACs during this period. Despite a national safety alert on the issue, a widespread rise in elevated INR test results was not found. Primary care has responded rapidly to changes in patient care during the COVID-19 pandemic.

\begin{tabular}{l} 
KEY QUESTIONS \\
WHAT IS ALREADY KNOWN ABOUT THIS \\
SUBJECT? \\
$\Rightarrow$ Since the beginning of the COVID-19 pandemic, the \\
National Health Service (NHS) has recommended \\
putting measures in place to reduce face-to-face \\
contact with patients where possible to reduce \\
the risk of transmission. This includes guidance to \\
consider prescribing a direct-acting oral anticoagu- \\
lant (DOAC) instead of warfarin in suitable patients. \\
However, it was not known how widespread this \\
practice was, or whether it results in potentially \\
dangerous coadministration. In addition, there has \\
one small report on reduced international nor- \\
malised ratio (INR) control during the pandemic. \\
WHAT DOES THIS STUDY ADD? \\
$\Rightarrow$ This is the first widespread study on the impact of \\
the NHS England guidance. It shows that a sub- \\
stantial number of patients in general practice in \\
England had their anticoagulant switched from war- \\
farin to a DOAC. It also shows that there was a small \\
number of patients who potentially received both \\
warfarin and a DOAC. We also showed that there \\
did not appear to be a widespread reduction in INR \\
control during the pandemic. \\
HOW MIGHT THIS IMPACT ON CLINICAL \\
PRACTICE? \\
\hline This study highlights that national prescribing guid- \\
ance can be implemented in primary care in England, \\
both on a large scale and at a fast pace when nec- \\
essary to improve patient care. It also highlights the \\
importance of good clinical governance processes \\
when switching medications, in order to reduce the \\
risk of coprescribing of both agents.
\end{tabular}




\section{BACKGROUND}

Anticoagulants are prescribed to people at risk of or for treatment of thromboembolism, which in some cases can result in stroke. Warfarin, a vitamin Kantagonist, has been the mainstay of oral anticoagulant treatment for decades. A patient's specific dose of warfarin is adjusted based on frequent blood tests that determine their international normalised ratio (INR), a measurement of blood clotting time. Factors such as changes in diet, alcohol intake, acute illness and concomitant medications can affect blood levels of warfarin and INR, requiring a temporary increase in frequency of testing. The quality of the anticoagulation control is assessed by the proportion of time in therapeutic range (TTR). Direct-acting oral anticoagulants' (DOACs; rivaroxaban, dabigatran etexilate, apixaban and edoxaban) mechanism of action does not alter the INR and therefore people taking DOACs require less frequent drug safety monitoring (eg, renal function). Nationally, the prescribing of DOACs has increased steadily since their recommendation by NICE for atrial fibrillation (AF) in 2012. ${ }^{1}$

Following the onset of the COVID-19 pandemic, the National Health Service (NHS) responded to deliver healthcare services in a manner that minimised risk of virus transmission. Most patients taking the anticoagulant warfarin require frequent blood tests, the INR test, potentially increasing their chance of exposure to SARSCoV-2. NHS England issued guidance in March $2020^{2}$ to support local NHS organisations to manage their anticoagulant services; this included identifying people suitable for switching from warfarin to DOACs. In May 2020, NHS England wrote to Clinical Commissioning Groups (CCGs), the local NHS bodies responsible for medicines use, advising that apixaban or rivaroxaban should be prescribed in place of warfarin for people able to change, ${ }^{3}$ following a procurement exercise that secured additional stock at reduced prices. In October 2020, the Medicines and Healthcare products Regulatory Authority (MHRA) issued a safety alert, warning about an increase in the number of people with substantially elevated INR levels observed during the pandemic and also warned that of some people for whom warfarin was inadvertently continued after switching to DOACs ${ }^{4}$; however, this document gave no indication of the scale of these problems.

Using a retrospective cohort study design, we set out to: evaluate the proportion and characteristics of prior warfarin users who switched to DOACs and how many subsequently reverted; identify potentially unsafe coprescribing of warfarin and DOACs; and measure the frequency of INR testing during the pandemic for people taking warfarin, any changes to TTR and the extent to which elevated INRs were observed. This was conducted as a 'proof of concept' for the use of the new OpenSAFELY analytics platform to rapidly understand service impacts during the COVID-19 pandemic and inform support for primary care.

\section{METHODS}

\section{Study design}

Prescribing and testing practice was analysed by conducting a retrospective cohort study using data from English NHS general practices.

\section{Data sources}

Primary care records managed by the General Practice (GP) software provider TPP were assessed using OpenSAFELY, a data analytics platform created by our team on behalf of NHS England to address urgent COVID-19 research questions (https://opensafely.org). OpenSAFELY provides a secure software interface allowing the analysis of pseudonymised primary care patient records from England in near real-time within the Electronic Health Record (EHR) vendor's highly secure data centre, avoiding the need for large volumes of potentially disclosive pseudonymised patient data to be transferred off-site. This, in addition to other technical and organisational controls, minimises any risk of reidentification. Similarly, pseudonymised datasets from other data providers are securely provided to the EHR vendor and linked to the primary care data. The dataset analysed within OpenSAFELY is based on 24 million people currently registered with GP surgeries using TPP SystmOne software $(40 \%$ of England's population). It includes pseudonymised data such as coded diagnoses, medications and physiological parameters but no free-text data. Further details on our information governance can be found under ethics approval.

\section{Data processing}

We extracted data for all people who were issued with a prescription or had an active repeat prescription for warfarin or DOACs between January 2019 and August 2020, and the relevant dates of each prescription and INR test-related activity. We used warfarin, DOAC and INR codelists from OpenSAFELY ${ }^{5}{ }^{6}$ to identify these activities, with 'high INRs' classified as INR of $>8 / \geq 8$ (as indicated) in line with thresholds relied on by the MHRA. ${ }^{7}$ We identified TTR using the following CTV3 codes 'YavzQ', '42QE2' (redundant) and 'Xaa68'.

\section{People switching from warfarin to DOACs following NHS England guidance}

We identified people issued a prescription for warfarin (but no DOACs) between December 2019 and February 2020 and assessed how many received at least one DOAC ('switched') in March-May 2020 or only received warfarin (figure 1). Of those switching, we counted how many later received warfarin again during this period ('switched back'). Of those remaining on warfarin, we counted how many received an INR test, a high INR value or had a TTR recorded. This analysis was repeated for the subsequent 3-month period (warfarin March-May 2020, switching June-August 2020) and for the corresponding periods in the previous year. Descriptive tables were generated to describe the cohort. 


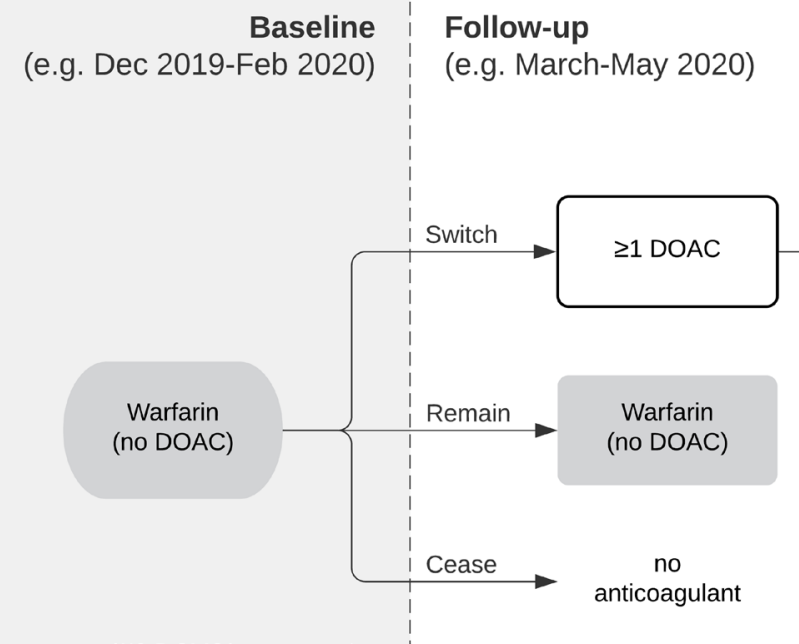

Figure 1 Identification of patients. Flow chart illustrating how patients switching from warfarin to DOACs (and those switching back within the same period) were identified based on prescriptions issued. DOACs, direct-acting oral anticoagulants.

We also assessed the overall rate of people starting new DOAC repeat prescriptions each month from January 2019 to August 2020 (with 'new' defined as people having no DOAC repeat prescription ending within the previous 3 months). We assessed how many of whom had switched from warfarin, defined as people having a warfarin repeat prescription with an end date in the previous 3 months. Figures were plotted on a time trend chart.

\section{Potentially unsafe coprescribing of warfarin and a DOAC}

For each month, January 2019-August 2020, patients were identified who: (A) were issued a prescription for DOAC and warfarin on the same day or (B) had a repeat medication initiated for both DOAC and warfarin on the same day (and also had at least one warfarin/DOAC prescription issued within the month). A repeat prescription allows items to be issued, therefore having active repeats for both medications could allow both warfarin and a DOAC to be issued to a patient in error (however, prescriptions can also be issued acutely without a repeat). We identified whether these people had at least one of these cancelled the same day. Figures were plotted on a time trend chart.

\section{INR tests during the COVID-19 period}

Each month, INR tests and TTR values were identified for people on warfarin (defined as people with warfarin issued within the previous 3 months, and no DOAC issued after the latest warfarin). We plotted the rate per 1000 warfarin patients (and for TTRs, also the rate per 1000 INR tests and the rate per 1000 people tested) on time trend charts.

\section{Factors associated with switching from warfarin to a DOAC during the pandemic}

We investigated factors associated with switching from warfarin to DOAC using mixed effects logistic regression. We included the variables in table 1 as factors in the model. We excluded ethnicity due to $\sim 30 \%$ missingness. We also planned to include the patient's practice, but this was later removed to allow the model to converge. The population was: people aged 18-110 years at the start of the follow-up period; registered with a single practice for the 6 months prior to the follow-up period (to ensure completeness of covariate data); prescribed warfarin at least once in the baseline period; first prescribed warfarin at least 6 months ago (to exclude very recent initiations); not prescribed a DOAC during the baseline period; and prescribed at least one DOAC or warfarin during the follow-up period (to exclude people who ceased treatment or died before receiving any prescription in this period). The outcome was the prescription of at least one DOAC during the follow-up period. Baseline period: 3 months prior to the national 'stay at home' order (16 December 2019-15 March 2020); follow-up period: the following 3 months (16 March 2020-15 June 2020).

\section{Software and reproducibility}

Data management was performed using Python V.3.8 and SQL, and regression analysis using Stata V.16.1. All codes for the OpenSAFELY platform, and for data management and analyses for this study, are available for inspection and reuse under open licenses on GitHub (https://github.com/opensafely/anticoagulant-switching-research). All codelists are available for inspection and reuse from https://codelists.opensafely.org.

\section{Patient and public involvement}

Patients were not formally involved in developing this specific study design that was developed rapidly in the context of a global health emergency. We have developed a publicly available website https://opensafely.org/ through which we invite any patient or member of the public to contact us regarding this study or the broader OpenSAFELY project. 
Table 1 Variables used to assess factors associated with switching (present on the first day of the follow-up period unless otherwise stated)

\begin{tabular}{|c|c|c|}
\hline Factor & Detailed information on coding & Codelist (where applicable) \\
\hline Indices of Multiple Deprivation (IMD) & Deciles based on residential postcode & Supplied by TPP. \\
\hline Diagnosis of AF & Yes/no; any time prior to follow-up period & $\begin{array}{l}\text { https://codelists.opensafely.org/codelist/ } \\
\text { opensafely/atrial-fibrillation/2020-07-09/ }\end{array}$ \\
\hline Renal function & $\begin{array}{l}\text { Latest estimated Glomerular Filtration Rate (eGFR } \\
\text { function) }(<30,30-59 \text { and } \geq 60 \text { ) in the } 12 \text { months to } \\
\text { the end of the baseline period (or 'no evidence' for } \\
\text { those with no creatinine recorded in this period). }\end{array}$ & $\begin{array}{l}\text { eGFR calculated from creatinine records. } \\
\text { Creatinine clearance is recommended renal } \\
\text { function assessment for tailoring DOAC doses } \\
\text { for individual people. Automated calculation of } \\
\text { Creatinine Clearance (CrCl) is not yet available } \\
\text { in OpenSAFELY, and eGFR is used as pragmatic } \\
\text { alternative. }\end{array}$ \\
\hline Recent renal function test & $\begin{array}{l}\text { Yes/no; within last } 4 \text { months and up to the end of the } \\
\text { study period (this conservatively allows tests slightly } \\
\text { outside of the recommended 3-month period, and any } \\
\text { time during the switching period). }\end{array}$ & $\begin{array}{l}\text { Creatinine test or: } \\
\text { ' } 451 \text { '. Renal function test. } \\
\text { 'XacUK' eGFR using creatinine (calculated } \\
\text { using Chronic Kidney Disease-Epidemiology } \\
\text { Collaboration (CKD-EPI) per } 1.73 \text { square } \\
\text { metres. }\end{array}$ \\
\hline Number of INR tests & $0,1-3,4-6$ or $7+$ tests during the baseline period & $\begin{array}{l}\text { https://codelists.opensafely.org/codelist/ } \\
\text { opensafely/international-normalised-ratio- } \\
\text { inr/2020-10-22 }\end{array}$ \\
\hline Previously prescribed DOACs* & Yes/no; any time prior to the baseline period. & $\begin{array}{l}\text { https://codelists.opensafely.org/codelist/ } \\
\text { opensafely/direct-acting-oral-anticoagulants- } \\
\text { doac/2020-10-05/ }\end{array}$ \\
\hline Explicitly recorded contraindicaton to DOAC & Yes/no; any time prior to the follow-up period. & $\begin{array}{l}\text { https://codelists.opensafely.org/codelist/ } \\
\text { opensafely/explicit-contraindication-to-doacs- } \\
\text { direct-acting-anticoagulants/2020-10-19/ }\end{array}$ \\
\hline
\end{tabular}

\footnotetext{
*The earliest actual date of an event may be missing due to incomplete patient records (eg, lost in transfer between practices), but the earliest recorded date can be used as an approximation.

AF, atrial fibrillation; CCGs, Clinical Commissioning Groups; DOAC, direct-acting oral anticoagulant; INR, international normalised ratio; NHS, National Health Service.
}

\section{RESULTS \\ Patients switching from warfarin to DOACs following NHS England advice}

A total of 164000 people were prescribed warfarin between December 2019 and February 2020, of whom $12.2 \%$ (20 000) were prescribed a DOAC between March and May 2020 (table 2). This was substantially higher than the previous year $(3.5 \%)$ and the following 3 months $(4.4 \%)$ (table 2). Of those who switched to a DOAC, $5.8 \%(\mathrm{n}=1200)$ also received a subsequent prescription for warfarin (switched back), compared with $4.1 \%$ the previous year (table 2). Of those remaining on warfarin, $80.1 \%$ had at least one INR test recorded during MarchMay compared with $83.7 \%$ the previous year, $38.6 \%$ had at least one recorded INR TTR $(37.9 \%$ the previous year), and $0.5 \%$ had a high INR $(\geq 8)$ result $(0.4 \%$ the previous year; table 2). During March-May 2020, edoxaban and apixaban were the most commonly selected DOACs among those who were switched $(38.1 \%$ and $33.5 \%$, respectively; table 3). During June-August 2020, 
Table 2 Warfarin patients switching to DOACs or remaining on warfarin

\begin{tabular}{|c|c|c|c|c|}
\hline \multirow[b]{2}{*}{ Period } & \multicolumn{4}{|c|}{ Patient count, thousands (percentage) } \\
\hline & March-May & & June-August & \\
\hline Year & 2020 & 2019 & 2020 & 2019 \\
\hline Baseline warfarin patients ${ }^{\star \star}$ & 164000 & 195000 & 143200 & 185900 \\
\hline Switched & $20000(12.2)$ & $6900(3.5)$ & $6300(4.4)$ & $5900(3.2)$ \\
\hline Continued warfarin & $136100(83.0)$ & $177300(90.9)$ & $128200(89.5)$ & $169900(91.4)$ \\
\hline No anticoagulants $†$ & $7900(4.8)$ & $10800(5.6)$ & $8700(6.1)$ & $10100(5.4)$ \\
\hline $\begin{array}{l}\text { Switched back } \\
\text { (\% of switchers) }\end{array}$ & $1200(5.8)$ & $300(4.1)$ & $300(4.7)$ & $300(4.4)$ \\
\hline $\begin{array}{l}\text { At least one INR } \\
\text { (\% of continued) }\end{array}$ & $109100(80.1)$ & $148400(83.7)$ & $101100(78.8)$ & $140000(82.4)$ \\
\hline $\begin{array}{l}\text { At least one TTR } \\
\text { (\% of continued) }\end{array}$ & $52600(38.6)$ & $67200(37.9)$ & $50300(39.2)$ & $66500(39.1)$ \\
\hline $\begin{array}{l}\text { High INR }(\geq 8) \\
\text { (\% of continued) }\end{array}$ & $700(0.5)$ & $700(0.4)$ & $400(0.3)$ & $600(0.3)$ \\
\hline
\end{tabular}

Number and percentage of warfarin patients who continued on warfarin, received a DOAC ('switched') or had no anticoagulants, during March-May 2020 compared with 2019 and similarly for June-August. Also showing the percentage of those switching to a DOAC who later received warfarin 'switched back' (within the same 3-month period); and the percentage of those remaining on warfarin who had at least one INR test, INR TTR or high INR ( $\geq 8)$ recorded. Patient counts are rounded to the nearest 100.

*Baseline warfarin patients for each period were those issued warfarin at least once (but no DOACs) in the 3 months immediately prior to the period shown.

†Those with no anticoagulants (referring to warfarin and DOACs only) in the follow-up period may have discontinued anticoagulant treatment, moved to a non-TPP practice, died or simply had a long period without a new prescription from their GP (eg, due to a hospital stay or longer than usual prescription duration).

DOACs, direct-acting oral anticoagulants; INR, international normalised ratio; TTR, time in therapeutic range.

apixaban had increased to $40.3 \%$, with edoxaban dropping to $33.4 \%$ (table 3 ).

The initiation of repeat prescriptions for DOACs to new patients increased $~ 1.5$-fold during March and April 2020 and subsequently dropped slightly below normal levels (figure 2). Much of this increase was attributable to people switching from warfarin $(40.3 \%$ in March $57.5 \%$ in April), compared with the normal rate of $\sim 15 \%$ per month (figure 2).

\section{Potentially unsafe coprescribing of warfarin and a DOAC}

Prior to the pandemic period, typically 50-100 people per month had both warfarin and a DOAC issued on the same day, rising sharply to 246 in April 2020 (0.06\% of all people receiving a DOAC or warfarin) before declining gradually to almost baseline by August 2020 (figure 3A). Only a small proportion of these patients had at least one of their coprescriptions cancelled on the same day (ie, prescription end date equal to start date). Prior to the pandemic, 60-110 patients per month had both warfarin and a DOAC repeat prescription initiated on the same day (figure 3B). This figure reached a peak of $\sim 170$ in April and declined rapidly to near-normal levels from May 2020. However, this rise was compensated by an increase in the number of repeat prescriptions that were cancelled the same day (usually warfarin).

\section{INR tests during the COVID-19 period}

Of 164000 people on warfarin prior to March 2020, $80.1 \%$ had an INR test recorded and $35 \%$ had a TTR

Table 3 Types of DOAC selected

\begin{tabular}{|c|c|c|c|c|c|c|c|c|c|c|}
\hline \multirow[b]{2}{*}{ Period } & \multirow[b]{2}{*}{ Year } & \multicolumn{2}{|l|}{ Apixaban } & \multicolumn{2}{|l|}{ Edoxaban } & \multicolumn{2}{|l|}{ Rivaroxaban } & \multicolumn{2}{|c|}{ Dabigatran etexilate } & \multirow[b]{2}{*}{ Total } \\
\hline & & Patient count & $\%$ & Patient count & $\%$ & Patient count & $\%$ & Patient count & $\%$ & \\
\hline \multirow[t]{2}{*}{ March-May } & 2020 & 6700 & 33.5 & 7620 & 38.1 & 5550 & 27.8 & 120 & 0.6 & 19990 \\
\hline & 2019 & 3510 & 51.1 & 1040 & 15.2 & 2190 & 31.9 & 120 & 1.8 & 6860 \\
\hline \multirow[t]{2}{*}{ June-August } & 2020 & 2550 & 40.3 & 2110 & 33.4 & 1620 & 25.6 & 40 & 0.6 & 6320 \\
\hline & 2019 & 2900 & 49.5 & 1110 & 18.9 & 1760 & 30 & 90 & 1.6 & 5860 \\
\hline
\end{tabular}

Number and percentage of warfarin patients switched to each of the four types of DOAC between March and May 2020 compared with 2019, and similarly for June-August, for people who were prescribed warfarin during the previous 3-month period. Patient counts are rounded to the nearest 10 . Percentages may not add to exactly 100 due to rounding.

DOAC, direct-acting oral anticoagulant. 
Patients with a new DOAC repeat prescription initiated, per month

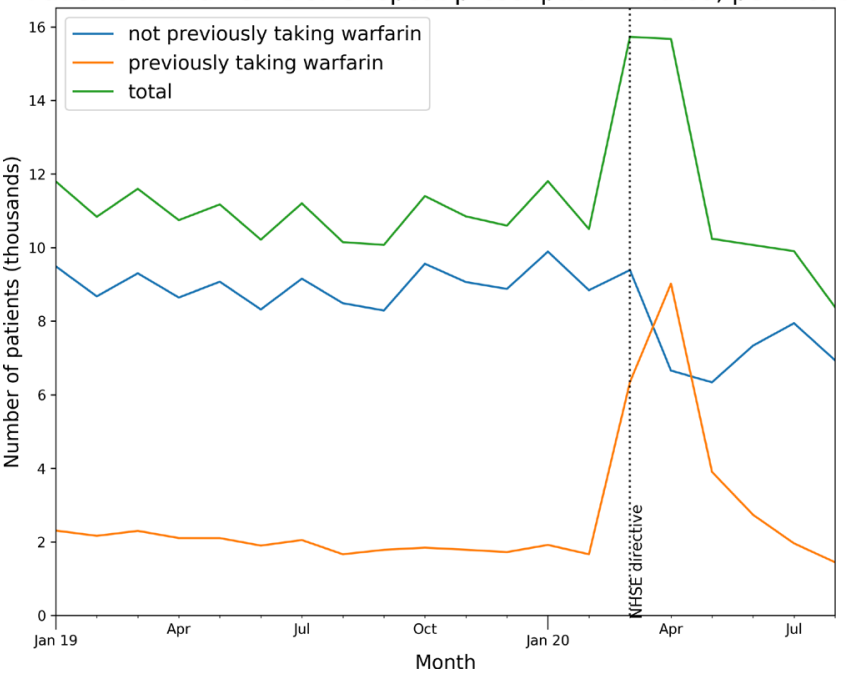

Figure 2 Patients newly initiated on DOAC repeats. Number of people having a new DOAC repeat prescription initiated per month (where the patient had no prior DOAC repeat ending within the previous 3 months), indicating whether or not patients had previously had a repeat prescription for warfarin (ending same month or within previous 3 months). DOAC, direct-acting oral anticoagulants.

recorded between March and May 2020 (table 2). The number of warfarin patients tested each month was approximately constant prior to the pandemic (589 per 1000 eligible patients per month, January 2019-March 2020) but with a small reduction during the pandemic (figure $4 \mathrm{~A}$ ). The monthly testing rate reduced by $14 \%$, to 506.8 (April-August 2020), a reduction of 82.4 per 1000 patients being tested per month $(14.0 \%$ reduction). The number of TTRs recorded followed a similar pattern (figure 4B), and where TTR was recorded, the mean value for those who were still on warfarin remained relatively constant (figure 4C). Figure 4D,E illustrate the rate of high INRs per 1000 warfarin patients, per 1000 INR tests and per 1000 patients tested. A small peak is observed in April, but the absolute number of high INR results $(\geq 8)$ was 470 , only slightly higher than January's figure of 420 (online supplemental table S1).

\section{Factors associated with switching from warfarin to a DOAC during the pandemic}

A total of 149243 people were included in this analysis (online supplemental table S3) and our logistic regression model results are presented in table 4 . People who had reduced renal function were less likely to switch from warfarin to DOAC compared with those with eGFR $\geq 60$ (eGFR <30: OR $0.51,95 \%$ CI 0.46 to 0.57 ; eGFR 30-59: OR $0.92,95 \%$ CI 0.88 to 0.96 ). Those with no eGFR in the last 12 months but who had had a renal function test recorded in the last 4 months were more likely to switch (OR 2.11, 95\% CI 2.02 to 2.21), while those with no eGFR or recent renal function test were less likely to switch $(0.40,95 \%$ CI 0.38 to 0.42$)$. People with multiple recent INR tests were more likely to switch (over seven vs none recorded, OR 1.88, 95\% CI 1.77 to 1.99); older people (over 75 vs under 65 , OR $1.89,95 \%$ CI 1.77 to 1.99 ); people living in a care home (OR 1.32, 95\% CI 1.15 to 1.51); and people with a diagnosis of AF (OR 1.95, 95\% CI 1.87 to 2.03). People with a recorded contraindication for a DOAC were less likely to be switched (OR 0.37, $95 \%$ CI 0.26 to 0.52). We also found that Sustainability and Transformation Partnership (STP) membership, when modelled as a random effect, was a significant driver of variation $(p<0.0001)$.
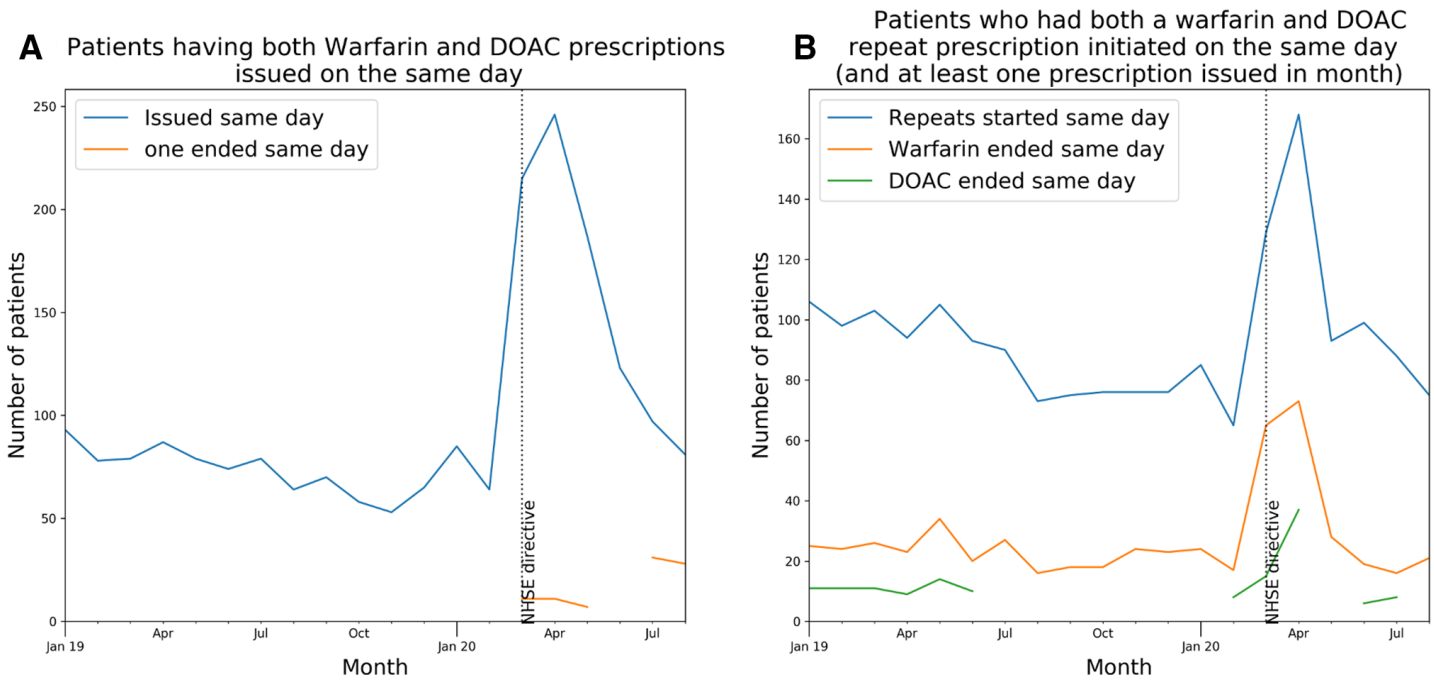

Figure 3 Patients coprescribed warfarin and a DOAC. Number of patients having (A) both warfarin and a DOAC prescription issued on the same day, also indicating how many patients for whom one of those prescriptions was cancelled the same day (ie, its end date was equal to its start date); (B) both warfarin and a DOAC repeat prescriptions initiated on the same day (restricted to patients who also had at least one warfarin or DOAC prescription issued in the given month), also indicating how many patients for whom one of those repeat prescriptions was cancelled the same day (ie, its end date was equal to its start date). Patient counts are shown in online supplemental table S2. DOAC, direct-acting oral anticoagulant. 


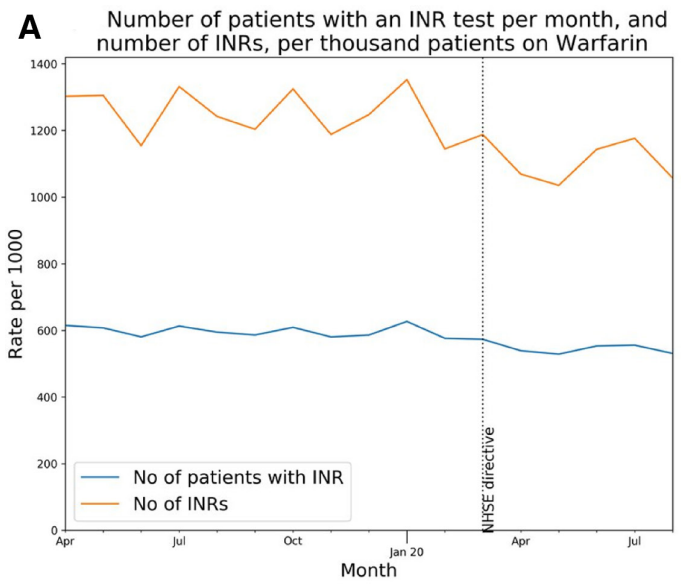

B Number of patients with a TTR recorded each month, and

number of TTRs, per thousand patients on warfarin

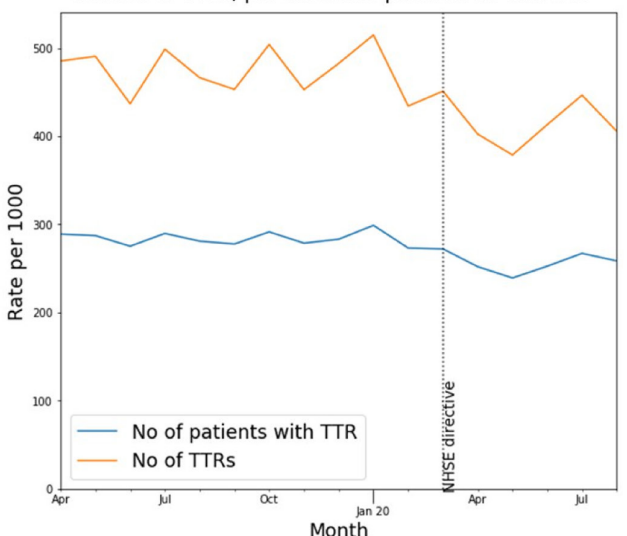

C

D
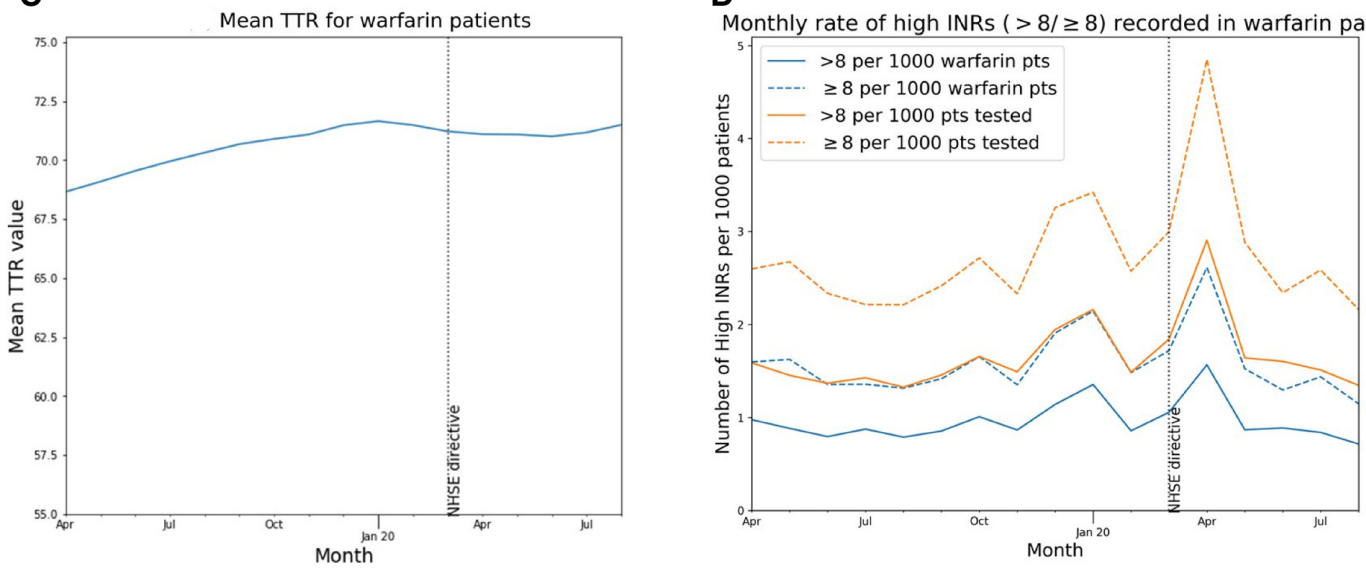

Figure 4 INR tests and recorded TTRs in warfarin patients. (A) Number of patients having an INR test, and the total number of INR tests carried out, per thousand warfarin patients. (B) Number of patients having a TTR recorded, and the total number of TTRs, per thousand warfarin patients. (C) Mean TTR value across warfarin patients tested. (D) Monthly rate of elevated INRs (as $>8$ and $\geq 8$ ) recorded in warfarin patients per thousand warfarin patients and thousand patients having an INR test. INR, international normalised ratio; TTRs, time in therapeutic range.

\section{DISCUSSION}

\section{Summary}

We observed a substantial increase in people switching from warfarin to a DOAC after NHS England advice to do so during the COVID-19 pandemic. We were able to identify in the data that a small but substantial number of people $(n=246,0.06 \%)$ simultaneously prescribed warfarin and a DOAC in April 2020 and were potentially at risk of serious adverse effects. Overall, the rate of INR testing for those on warfarin dropped by $14 \%$, although we observed no substantial change in the proportion of INR tests that reported a clotting time outside the desired range. Factors associated with switching from warfarin to DOACs included older age, higher number of recent INR tests, diagnosis of AF, normal renal function and care home residency.

\section{Strengths and weaknesses}

The key strength of this study is the scale, timeliness and completeness of the underlying data. The OpenSAFELY platform runs analyses across the full, raw, single-eventlevel medical records of all patients at $40 \%$ of all GP practices in England, including all tests, treatments, diagnostic events and other salient clinical and demographic information. We also recognise some limitations. We assessed the number of prescriptions issued or initiated on repeat prescriptions. We cannot currently access information on which medications were dispensed. An individual appearing to receive warfarin and a DOAC may therefore not receive, or take, both medications: for example, a doctor might notice the coprescribing some time after the consultation and cancel the prescription, or a pharmacist may decline to dispense both medications for safety reasons. Finally, a patient may be informed not to take the medicine by their healthcare professional even if they received a dispensed medicine. Nonetheless, this does not diminish the finding that coprescribing of warfarin and DOACs occurs and that incidence increased substantially during COVID-19 to over 200 people in 1 month. Another limitation relates to missing INR data: people in England often have their warfarin managed by an 'anticoagulation clinic' in a community service, hospital outpatient department or neighbouring general practice with specialist expertise; these clinics typically use bespoke software to record all relevant results related to INR, which may not be transferred in structured data into 
Table 4 Factors associated with switching from warfarin to a DOAC during the pandemic

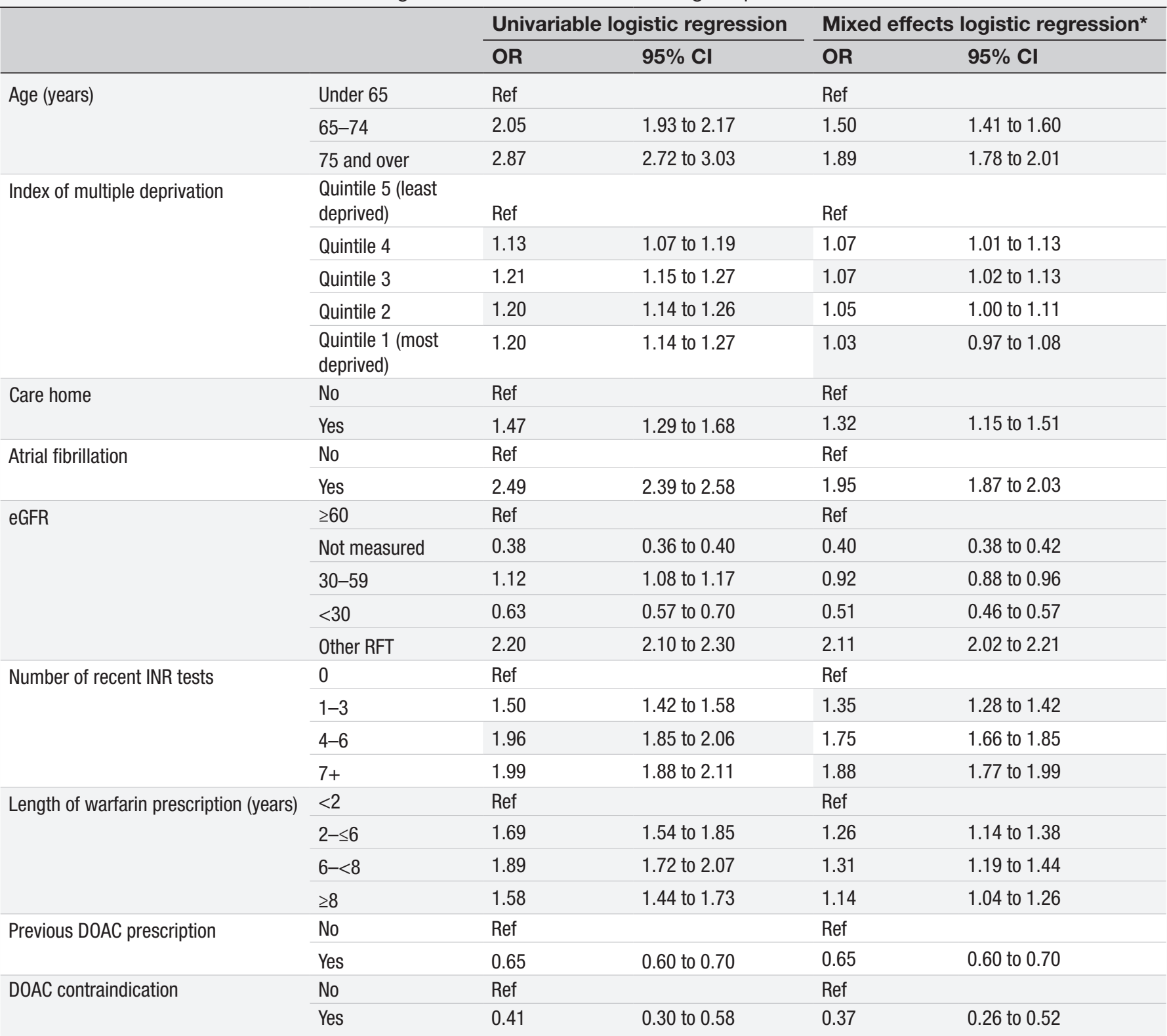

Patient counts are reported in online supplemental table S3.

${ }^{*}$ Adjusted for the variables in the table STP membership as a random effect.

DOAC, direct-acting oral anticoagulant.

GP records but would be visible to clinicians delivering direct care for a patient; this could explain the apparent absence of INR tests for some people but not negatively impact clinical care. Our regression analysis could not capture all possible causes of confounding; in particular. the patient's practice was excluded to allow the model to converge, and this would be expected to have a strong influence on anticoagulant prescribing.

\section{Findings in context}

A recent systematic review of healthcare utilisation during the pandemic found that utilisation reduced by approximately one-third during the pandemic, ${ }^{8}$ but we are not aware of any published studies assessing switching of anticoagulant therapy during the pandemic or implementation of NHS England anticoagulation guidance. We found that edoxaban and apixaban were favoured DOACs when switching from warfarin, not wholly in line with NHS England advice from May $2020,{ }^{3}$ which recommends apixaban and rivaroxaban. In October, NICE published draft guidance on AF recommending apixaban and dabigatran; however, we found dabigatran was prescribed in a very small proportion of switches. ${ }^{9}$ In England, general practitioners have autonomy in the selection of treatments they prescribe but are obliged to consider a wide number of factors that may influence choice of DOACs. ${ }^{10}$ Our previous research has shown NHS England guidance advising GPs to stop 
prescribing certain medicines that did not cause an immediate noticeable change in prescribing patterns, ${ }^{11}$ and CCG membership has a strong association with GP prescribing choices. ${ }^{12-15}$ Our study shows evidence of switching after the guidance and although we were not able to directly assess CCG membership, our study did find that STPs, an administrative region made up of one or more CCGs, were a significant driver of variation. The MHRA safety alert on anticoagulation identified a 'small number of patients' coprescribed warfarin and a DOAC but provided no further information on the scale of the problem. One Swiss hospital found $0.8 \%$ of its patients on anticoagulant therapy $(121 / 15812)$ were coprescribed two anticoagulants in a single year. ${ }^{16}$ However, $88.7 \%$ of these cases involved coprescription of DOACs and low-molecular weight heparins, not included in our study. As regards INR tests, the MHRA reports appears to have been triggered by a root cause analysis from a single centre in London, ${ }^{17}$ reporting that between 1 March and 17 April 2020, $0.9 \%$ (30/3214) of INRs were high (>8.0) compared with $0.1 \%(6 / 4079)$ the previous year. Analysing the full records of $40 \%$ of patients in England, we found only a small peak in high INRs and with no obvious change in the mean TTR recorded for those still on warfarin.

\section{Policy implications for research and practice}

The COVID-19 pandemic has brought new challenges for the NHS to deliver safe and effective routine care. NHS England issued anticoagulant guidance at the peak of the pandemic and a substantial number of people were switched in line with this guidance. This study using OpenSAFELY demonstrates it is possible to use routinely collected raw EHR data on a specific clinical area to support evaluation of national guidance and safety alerts during the COVID-19 pandemic. There is a need for high quality applied practical research to support healthcare organisations' responses to the pandemic; OpenSAFELY can be used by NHS England, the MHRA and others such as NICE to rapidly assess in near-real time the impact of policy and clinical guidance as well as informing future versions of guidance. Specific areas of focus for anticoagulants during COVID-19 could include: identifying people eligible for switching who have not switched yet; assessing the scale of clinical work needed; evaluating completeness of uptake; prioritising risk groups; understanding causes of coprescription of warfarin and DOACs; evaluating variation in organisations response to new guidance $^{14}$; and better understanding the factors identified by MHRA as being associated with elevated INRs such as coprescribing of antibiotics. ${ }^{4}$

\section{Summary}

We observed increased switching of anticoagulants from warfarin to DOACs at the outset of the COVID-19 pandemic in England. We did not find a widespread rise in elevated INR test results that may be reassuring after a recent MHRA safety alert, although we did observe a small but substantial number of people who were coprescribed warfarin and DOACs.
Author affiliations

${ }^{1}$ The DataLab, Nuffield Department of Primary Care Health Sciences, University of Oxford, Oxford, UK

${ }^{2}$ TPP, Leeds, UK

${ }^{3}$ Department of Non-communicable Disease Epidemiology, London School of Hygiene \& Tropical Medicine, London, UK

${ }^{4}$ Department of Medical Statistics, London School of Hygiene \& Tropical Medicine, London, UK

${ }^{5}$ Department of Infectious Disease Epidemiology, London School of Hygiene \& Tropical Medicine, London, UK

${ }^{6}$ Population Health Sciences, Bristol Medical School, University of Bristol, Bristol, UK

Acknowledgements We are very grateful for all the support received from the TPP Technical Operations team throughout this work and for generous assistance from the information governance and database teams at National Health Service (NHS) England/NHSX.

Contributors Conceptualisation: HJC, BM, AJW and RC; data curation: HJC, AJW, $\mathrm{SB}, \mathrm{GH}, \mathrm{PI}, \mathrm{CB}, \mathrm{DE}$ and SD; formal analysis: HJC and AJW; funding acquisition: FH, LS and BG; investigation: HJC, BM, AJW, RC and BG; methodology: HJC, BM, AJW and RC; project administration: HJC, BM and RC; resources: HJC, SB, DE, FH and BG; software: HJC, AJW, RC, SB, GH, PI, DE, JC, SD and SH; supervision: BG; visualisation: HJC and AJW; writing - original draft: HJC, BM, AJW, RC, AM, AYSW and $B G$; writing - review and editing: all authors; $B G$ is guarantor.

Funding The OpenSAFELY platform is supported by funding from UKRI/MRC, Wellcome trust and funding from the National Core Studies programme. TPP provided technical expertise and infrastructure within their data centre pro bono in the context of a national emergency.

Disclaimer Funders had no role in the study design, collection, analysis and interpretation of data; in the writing of the report; and in the decision to submit the article for publication. The views expressed are those of the authors and not necessarily those of the NIHR, NHS England, Public Health England or the Department of Health and Social Care.

Competing interests All authors have completed the ICMJE uniform disclosure form at www.icmje.org/coi_disclosure.pdf and declare the following: over the past 5 years, BG has received research funding from the Laura and John Arnold Foundation, the NHS National Institute for Health Research (NIHR), the NIHR School of Primary Care Research, the NIHR Oxford Biomedical Research Centre, the Mohn-Westlake Foundation, NIHR Applied Research Collaboration Oxford and Thames Valley, the Wellcome Trust, the Good Thinking Foundation, Health Data Research UK (HDRUK), the Health Foundation and the World Health Organisation; he also receives personal income from speaking and writing for lay audiences on the misuse of science. LS reports institutional grants from Wellcome, Medical Research Council (MRC), NIHR andUK Research and Innovation (UKRI). LT reports grants from Wellcome and MRC. KB holds a Sir Henry Dale fellowship jointly funded by Wellcome and the Royal Society (107731/Z/15/Z). RME holds grants from NIHR, MRC and HDRUK. RM holds a Sir Henry Wellcome Fellowship funded by the Wellcome Trust (201375/Z/16/Z). HF holds a UKRI fellowship. IJD has received unrestricted research grants and holds shares in GlaxoSmithKline.

Patient consent for publication Not applicable.

Ethics approval NHS England is the data controller; TPP are the data processors, and the key researchers on OpenSAFELY are acting on behalf of NHS England. This implementation of OpenSAFELY is hosted within the TPP environment, which is accredited to the ISO 27001 information security standard and (is) NHS IG Toolkit compliant; ${ }^{18} 19$ patient data have been pseudonymised for analysis and linkage using industry standard cryptographic hashing techniques; all pseudonymised datasets transmitted for linkage onto OpenSAFELY are encrypted; access to the platform is via a virtual private network (VPN) connection, restricted to a small group of researchers; the researchers hold contracts with NHS England and only access the platform to initiate database queries and statistical models; all database activity is logged; only aggregate statistical outputs leave the platform environment following best practice for anonymisation of results such as statistical disclosure control for low cell counts. ${ }^{20}$ The OpenSAFELY research platform adheres to the data protection principles of the UK Data Protection Act 2018 and the EU General Data Protection Regulation 2016. In March 2020, the Secretary of State for Health and Social Care used powers under the UK Health Service (Control of Patient Information) Regulations 2002 to require organisations to process confidential patient information for the purposes of protecting public health, providing healthcare services to the public and monitoring and managing the COVID-19 
outbreak and incidents of exposure; this sets aside the requirement for patient consent. ${ }^{21}$ Taken together, these provide the legal bases to link patient datasets on the OpenSAFELY platform. GP practices, from which the primary care data are obtained, are required to share relevant health information to support the public health response to the pandemic and have been informed of the OpenSAFELY analytics platform. This study was approved by the Health Research Authority (REC reference 20/L0/0651) and by the London School of Hygiene \& Tropical Medicine SHTM Ethics Board (reference 21863).

Provenance and peer review Not commissioned; externally peer reviewed.

Data availability statement Data are available in a public, open access repository. Data management was performed using Python 3.8 and SQL, and regression analysis using Stata 16.1. All code for the OpenSAFELY platform, and for data management and analyses for this study, are available for inspection and reuse under open licenses on GitHub (https://github.com/opensafely/anticoagulantswitching-research). All codelists are available for inspection and re-use from https://codelists.opensafely.org/.

Open access This is an open access article distributed in accordance with the Creative Commons Attribution 4.0 Unported (CC BY 4.0) license, which permits others to copy, redistribute, remix, transform and build upon this work for any purpose, provided the original work is properly cited, a link to the licence is given, and indication of whether changes were made. See: https://creativecommons.org/ licenses/by/4.0/.

\section{ORCID iDs}

Helen J Curtis http://orcid.org/0000-0003-3429-9576

Brian MacKenna http://orcid.org/0000-0002-3786-9063

Alex J Walker http://orcid.org/0000-0003-4932-6135

Richard Croker http://orcid.org/0000-0002-8114-9186

Amir Mehrkar http://orcid.org/0000-0002-2098-1278

Caroline Morton http://orcid.org/0000-0003-0783-0042

Seb Bacon http://orcid.org/0000-0002-6354-3454

Chris Bates http://orcid.org/0000-0003-0113-2593

David Evans http://orcid.org/0000-0002-1100-079X

Jonathan Cockburn http://orcid.org/0000-0002-4681-4873

Krishnan Bhaskaran http://orcid.org/0000-0001-5364-8757

Anna Schultze http://orcid.org/0000-0002-1637-837X

Christopher T Rentsch http://orcid.org/0000-0002-1408-7907

William Hulme http://orcid.org/0000-0002-9162-4999

Laurie Tomlinson http://orcid.org/0000-0001-8848-9493

Rosalind M Eggo http://orcid.org/0000-0002-0362-6717

Angel Yun Wong http://orcid.org/0000-0002-8618-7333

Harriet Forbes http://orcid.org/0000-0001-6888-2212

lan Douglas http://orcid.org/0000-0002-8970-1406

Liam Smeeth http://orcid.org/0000-0002-9168-6022

Ben Goldacre http://orcid.org/0000-0002-5127-4728

\section{REFERENCES}

1 The DataLab. Direct oral anticoagulants (DOACs) measure. OpenPrescribing. Available: https://openprescribing.net/measure/ doacs/national/england/ [Accessed 30 Sep 2020].

2 NHS England. Clinical guide for the management of anticoagulant services during the coronavirus pandemic, 2020. Available: https:// web.archive.org/web/20200513044959/https://www.england.nhs.uk/ coronavirus/wp-content/uploads/sites/52/2020/03/C0077-Specialtyguide_Anticoagulant-services-and-coronavirus-v1-31-March.pdf [Accessed 20 Jul 2020].

3 NHS England. Supply of additional direct oral anticoagulants (DOACs) during COVID-19, 2020. Available: https://www.worcslmc. co.uk/cache/downloads/C0517-DOAC-briefing-for-CCGs_27-May. pdf

4 Warfarin and other anticoagulants - monitoring of patients during the COVID-19 pandemic. Available: https://www.gov.uk/government/ publications/warfarin-and-other-anticoagulants-monitoring-ofpatients-during-the-covid-19-pandemic/warfarin-and-other- anticoagulants-monitoring-of-patients-during-the-covid-19pandemic [Accessed 16 Oct 2020].

5 Curtis HJ, MacKenna B, Croker R. OpenSAFELY Codelists: direct acting oral anticoagulants (DOAC), 2020. Available: https:// codelists.opensafely.org/codelist/opensafely/direct-acting-oralanticoagulants-doac/2020-10-05/ [Accessed 28 Oct 2020].

6 Curtis HJ, MacKenna B, Croker R. OpenSAFELY Codelists: warfarin, 2020. Available: https://codelists.opensafely.org/codelist/opensafely/ warfarin/2020-10-05/ [Accessed 28 Oct 2020].

7 OpenSAFELY Codelists: international normalised ratio (Inr). Available: https://codelists.opensafely.org/codelist/opensafely/internationalnormalised-ratio-inr/2020-10-22/ [Accessed 3 Nov 2020]

8 Moynihan R, Sanders S, Michaleff ZA. Pandemic impacts on healthcare utilisation: a systematic review. medRxiv 2020.

9 Consultation | atrial fibrillation: management | guidance. NICE. Available: https://www.nice.org.uk/guidance/indevelopment/gidng10100/consultation/html-content-2 [Accessed 28 Oct 2020]

10 NHS England. Standard general medical services contract. Available: https://www.england.nhs.uk/gp/investment/gp-contract/

11 Walker AJ, Bacon S, Curtis $\mathrm{H}$, et al. Six months on: NHS England needs to focus on Dissemination, implementation and audit of its low-priority initiative. J R Soc Med 2019;112:4-5.

12 Walker AJ, Curtis HJ, Bacon S, et al. Trends, geographical variation and factors associated with prescribing of gluten-free foods in English primary care: a cross-sectional study. BMJ Open 2018;8:e021312.

13 Croker R, Walker AJ, Goldacre B. Why did some practices not implement new antibiotic prescribing guidelines on urinary tract infection? a cohort study and survey in NHS England primary care. J Antimicrob Chemother 2019;74:1125-32.

14 Walker AJ, Pretis F, Powell-Smith A, et al. Variation in responsiveness to warranted behaviour change among NHS clinicians: novel implementation of change detection methods in longitudinal prescribing data. BMJ 2019;367:15205.

15 MacKenna B, Curtis HJ, Walker AJ, et al. Trends and variation in unsafe prescribing of methotrexate: a cohort study in English NHS primary care. Br J Gen Pract 2020;70:e481-e488.

16 Rahmanzade R, Cabrera Diaz F, Zaugg C, et al. Therapeutic duplication of anticoagulants: a retrospective study of frequency and consequences in a tertiary referral hospital. Thromb J 2020;18:14.

17 Speed V, Patel RK, Byrne R, et al. A perfect storm: root cause analysis of supra-therapeutic anticoagulation with vitamin $\mathrm{K}$ antagonists during the COVID-19 pandemic. Thromb Res 2020;192:73-4.

18 Beta - data security standards. NHS digital. Available: https://digital. nhs.uk/about-nhs-digital/our-work/nhs-digital-data-and-technologystandards/framework/beta-data-security-standards [Accessed 30 Apr 2020].

19 Data security and protection toolkit. NHS digital. Available: https:// digital.nhs.uk/data-and-information/looking-after-information/datasecurity-and-information-governance/data-security-and-protectiontoolkit [Accessed 30 Apr 2020].

20 ISB1523: Anonymisation standard for publishing health and social care data. NHS digital. Available: https://digital.nhs. uk/data-and-information/information-standards/informationstandards-and-data-collections-including-extractions/ publications-and-notifications/standards-and-collections/isb1523anonymisation-standard-for-publishing-health-and-social-caredata [Accessed 30 Apr 2020].

21 Secretary of State for Health and Social Care - UK Government. Coronavirus (COVID-19): notification to organisations to share information, 2020. Available: https://web.archive.org/web/ 20200421171727/https://www.gov.uk/government/publications/ coronavirus-covid-19-notification-of-data-controllers-to-shareinformation

22 NICE. Overview | atrial fibrillation: management | guidance. Available: https://www.nice.org.uk/guidance/cg180 [Accessed 30 Sep 2020].

23 NICE. Nice key therapeutic topic: anticoagulants, including directacting oral anticoagulants (DOACs), 2016. Available: https://www. nice.org.uk/advice/ktt16/resources/anticoagulants-includingdirectacting-oral-anticoagulants-doacs-pdf-58757956094149 [Accessed 30 Sep 2020]. 